\title{
La programmation architecturale et urbaine. Émergence et évolutions d'une fonction
}

Architectural and Urban Programming. Emergence and Evolution of a Function

Jodelle Zetlaoui-Léger

\section{(2) OpenEdition}

\section{Journals}

Édition électronique

URL : http://journals.openedition.org/crau/312

DOI : $10.4000 /$ crau. 312

ISSN : 2547-5746

Éditeur

Éditions du patrimoine

Édition imprimée

Date de publication : 1 décembre 2009

Pagination : 143-158

ISBN : 978-2-85822-944-4

ISSN : 1296-4077

Référence électronique

Jodelle Zetlaoui-Léger, « La programmation architecturale et urbaine. Émergence et évolutions d'une fonction ", Les Cahiers de la recherche architecturale et urbaine [En ligne], 24/25 | 2009, mis en ligne le

01 septembre 2017, consulté le 19 avril 2019. URL : http://journals.openedition.org/crau/312 ; DOI :

$10.4000 /$ crau.312 
Après une clarification des significations associées aux termes de programme et de programmation, la contribution de J. ZetlaouiLéger met ici l'accent sur quatre des phénomènes qui ont marqué l'émergence et l'évolution de la programmation: I'apparition du programme comme mode de contrôle et de contractualisation dans l'acte de bâtir ; la singularisation de l'activité de programmation urbaine au cours des années 1960; l'autonomisation de la programmation par rapport à la conception, avec la réforme de l'ingénierie publique; l'effacement de la fonction de programmation urbaine à partir du milieu des années 1980 et sa recomposition récente autour de nouveaux enjeux. L'auteur s'attarde également sur les premières tentatives de théorisation de l'activité programmatique.

\section{La programmation architecturale et urbaine} Émergence et évolutions d'une fonction

JODELLE ZETLAOUI-LÉGER

L'examen de la production scientifique consacrée à la programmation architecturale et urbaine en France montre que très peu de travaux ont été consacrés à cette fonction, à la différence de ce que l'on peut observer dans les pays anglo-saxons, où elle a fait l'objet de nombreuses recherches et expérimentations des années 1950 à 1990 à l'initiative du Design Methods Movement ${ }^{1}$. Ce constat peut paraître paradoxal car l'autonomisation de cette activité est souvent présentée comme une spécificité française. La programmation a en effet été instituée comme une obligation réglementaire depuis trente ans par les décrets qui encadrent l'ingénierie publique, et elle est aujourd'hui prise en charge par des experts patentés.

À l'occasion d'une récente recherche sur la professionnalisation de cette fonction, nous avons été amenés à nous intéresser aux conditions de sa singularisation dans l'acte de bâtir ${ }^{2}$. Nous avons été rapidement confrontés aux multiples acceptions et aux différentes démarches qui lui ont été associées, au-delà du sens commun qui conduit nombre de professionnels de l'aménagement et de la construction à affirmer que " tout le monde programme et a toujours programmé ». Si l'on cherche à identifier les premières références à la

1. Le Design Methods Movement a été créé au début des années 1960 par des théoriciens-praticiens anglo-saxons en architecture, sur la base d'une critique des approches traditionnelles du processus de conception jugées trop intuitives et inopérantes face à des projets de plus en plus complexes à réaliser. Leur ambition initiale était de refonder la logique du processus de design par une réflexion sur la programmation comme méthode de résolution de problèmes permettant de mieux préparer le travail de mise en forme spatiale. Ce mouvement a donné lieu à deux courants. L'un, décliné des premières théories de Christopher Alexander, assimile la démarche de conception architecturale à un processus scientifique et technologique visant la recherche de la meilleure adéquation entre forme et fonction, grâce à la puissance de l'outil informatique; l'autre, proche des 
notion de programmation dans l'histoire de la production architecturale et urbaine en France, c'est en fait au mot programme que l'on se trouve généralement confronté. Le dictionnaire précise également l'antériorité de ce terme. Programme vient du grec programma et signifie étymologiquement « ce qui est écrit à l'avance ». Ce mot aurait été officiellement intégré à la langue française au XVII siècle, alors que celui de programmation I'aurait été au XIX siècle, avec le développement de la pensée rationaliste. Dans le sens commun, le programme détaille ce qui va être organisé.

En urbanisme et en architecture, on constate qu'il prend cependant au moins deux acceptions : il peut désigner la fonction générale ou la destination sociale de l'espace ou de l'édifice qui va être aménagé, ou correspondre à un texte donnant des instructions pour réaliser cette opération. Rarement employée jusqu'au milieu du $X x^{e}$ siècle bien que correspondant à une activité déjà mise en pratique, voire théorisée dans le domaine qui nous intéresse, la notion de programmation renvoie de façon générique à une démarche rationnelle décrivant des actions à mener. Elle évoque aussi l'idée de normalisation des "process ». Après la Seconde Guerre mondiale, elle prend une autre connotation plus stratégique comme démarche de résolution de problèmes ou d'aide à la décision. Ses principes, basés sur des activités de diagnostic, de scénarisation et d'évaluation, sont dans un premier temps définis et appliqués dans le cadre de la planification économique et dans celui du management des organisations en France et aux États-Unis, avant d'être étendus à l'aménagement de l'espace. Enfin, nous sommes en présence d'une autre signification, plus technico-administrative, qui est aujourd'hui la plus communément répandue en France car officialisée par la réforme de l'ingénierie publique au cours des années 1970 et 1980 : la programmation correspond à un moment du processus de projet de production d'études dites préalables ou de définition des objectifs, des contraintes et des besoins, aboutissant à l'élaboration d'un programme-cahier des charges précédant une phase de formalisation spatiale appelée conception.

Ces acceptions sont apparues à différentes périodes sans qu'elles se soient substituées les unes aux autres, conférant à chacun des termes de programme et de programmation un caractère polysémique. L'objet de cet article sera de faire le point sur les conditions d'émergence et d'évolution de la fonction de programmation dans le domaine de la production architecturale et urbaine en France, en nous centrant sur quelques phénomènes qui nous ont semblé décisifs dans ces transformations.

\section{Le programme comme mode de contrôle et de contractualisation dans l'acte de bâtir}

Bien que les termes de programme et de programmation soient officiellement apparus tardivement dans la langue française, les sens dont ils sont porteurs les ont précédés, y compris en architecture et en urbanisme. L'idée de programme comme document donnant des prescriptions relatives à un ouvrage à réaliser trouve sa concrétisation dès l'Antiquité, dans les civilisations grécoromaines. Il s'apparente alors à un devis descriptif permettant au pouvoir politique de s'assurer, lors de la conception d'édifices publics, du respect par les environmental studies, envisage la programmation comme une démarche heuristique, support à des pratiques « délibératives » dans le « process » de design. Les travaux du Design Methods Movement restent très méconnus en France, à l'exception de ceux d'Alexander, qui ont fait l'objet de traductions. Or, d'autres figures ont marqué ce mouvement, comme Horst Rittel, Henry Sanoff et Wolfgang Preiser. Parmi les premiers travaux, ceux qui ont été réalisés par William Peña et Gerald Davies avaient néanmoins influencé la démarche programmatique mise au point à l'occasion de la réalisation du Centre Georges Pompidou à Paris par François Lombard, un ingénieur-architecte qui revenait d'un séjour en Amérique du Nord. Cette expérience servira de référence pour la réforme de l'ingénierie publique. Les travaux du Design Methods Movement inspireront aussi les recherches menées sur la conception architecturale par Michel Conan et Robert Prost à la fin des années 1980 et au début des années 1990.
2. D'après Jacques Allégret, Nathalie Mercier et Jodelle Zetlaoui-Léger, L'Exercice de la programmation architecturale et urbaine en France, recherche menée dans le cadre du programme de recherche du réseau Ramau, Puca, 2005, 2 volumes. 
architectes ou les maçons de l'utilisation de certaines règles constructives et des coûts d'exécution prévus. Telle qu'elle commence à être utilisée en France à partir du XVIIe siècle, la notion de programme exprime la destination ou la vocation générale de l'espace à concevoir. Elle implique aussi toujours des contraintes à respecter, comme l'illustrent les plans-programmes des places royales, et renvoie à un contrat passé entre le client et son architecte, voire à un règlement de concours. Le programme, qui s'impose donc dès lors comme un outil de contrôle technique et administratif de l'acte de bâtir, s'est aussi rapidement révélé un formidable moyen de reproduction d'un ordre social.

La première grande entreprise de ce type sera, en Europe occidentale, le fait du pouvoir religieux. Dans Les Pierres sauvages ${ }^{3}$, roman historique retraçant la construction de l'abbaye du Thoronet au X XI ${ }^{e}$ siècle, dans le Sud de la France, Fernand Pouillon autorise son héros et narrateur, le « maître d'œuvre » chargé de la réalisation de cet édifice, à utiliser le terme de programme. Le mot évoque les différents éléments qui constitueront le futur ensemble architectural (le cloître, l'église, le dortoir, la bibliothèque...), ainsi que leur mode d'organisation spatiale. Ce programme constructif n'a manifestement pas d'existence matérielle. En revanche, il est bien présent dans l'esprit du maître d'œuvre. Au cours du récit, on comprend en effet progressivement que le programme et la règle monastique, en l'occurrence celle de saint Benoît, qui devra guider la vie des moines de la future communauté, sont en fait confondus ${ }^{4}$. La plupart des abbayes cisterciennes ont été réalisées sur la base d'un schéma d'organisation programmatique que défi- nira quelques années plus tard Bernard de Clairvaux, fondateur de l'ordre, à partir d'un travail d'interprétation des préceptes de cette Règle. Pour s'assurer du respect de la discipline religieuse de saint Benoît dans tous les monastères de l'ordre, saint Bernard exprimera ainsi la traduction spatiale des différents rituels devant rythmer la vie de la communauté. Partout en France, les abbayes cisterciennes devront donc comporter les mêmes lieux, offrir la même organisation spatiale et présenter les mêmes canons stylistiques pour que s'accomplisse de façon uniforme la Règle. Cette structuration de l'espace dictée par le temps, inscrite en filigrane dans la règle de saint Benoît, fait de celle-ci une sorte de programme architectural voire urbain, bien qu'elle ne parle pas toujours directement d'espace. Elle guide la réalisation de petites cités fermées qui contribueront à pérenniser une communauté et un projet de vie qui perpétueront l'esprit de la Règle.

Le programme d'un édifice apparaît comme un moyen de transmettre des valeurs, mais plus encore, de reproduire un ordre social. Cette logique inspirera aux XVIII et XIXe siècles les grands corps de l'État en France dans leur double préoccupation d'affirmation d'une identité nationale et de maintien d'un contrôle social à travers une production massive et normalisée d'équipements collectifs ${ }^{5}$. Le programme s'impose en effet à partir de cette période comme un outil permettant de diriger au niveau central l'équipement du territoire national en maîtrisant aussi bien les produits que les " process ». II organise un mode de contractualisation entre l'État, les autorités locales et les maîtres d'œuvre. Enfin, il réglemente les conditions de subventions aux organismes
3. Fernand Pouillon, Les Pierres sauvages, Paris, Le Seuil, 1964.

4. Rappelons que le terme d'ecclesia, qui signifie en grec assemblée, a donné en français le mot église, ce dernier désignant à la fois un bâtiment et une organisation.
5. Normaliser vise à déterminer une référence et un modèle pour un objet ou une activité. La normalisation permet la standardisation et l'uniformisation des tâches, facilite la reproductibilité des procédures, tout en ayant vocation à assurer un principe d'équité et de justice dans l'action de l'institution, en l'occurrence dans celle de l'État. François Fourquet et Lion Murard, citant Georges Canguilhem dans « Les équipements du pouvoir » (Recherches, n० 13, 1973, p. 155-156), soulignent que le terme de normal serait apparu en 1759 et celui de normalité en 1834. 


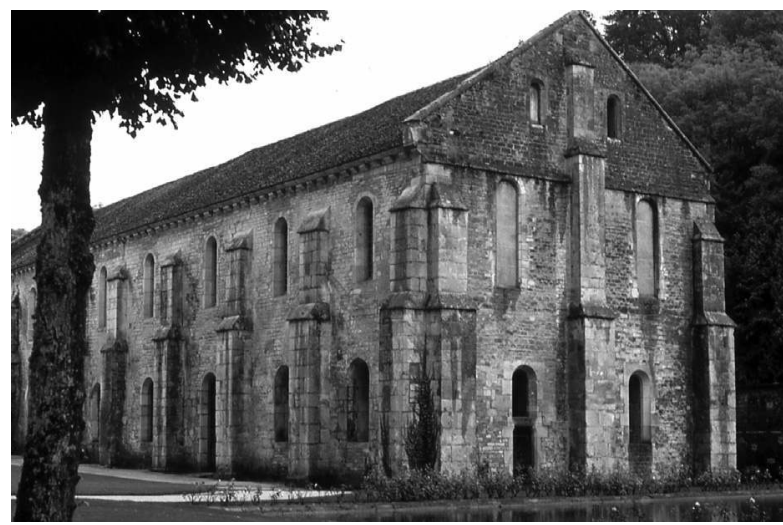

L'abbatiale

et le cloître.

J. Zetlaoui-Léger.

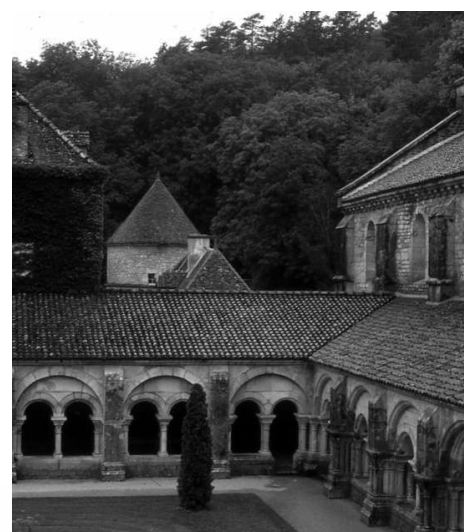

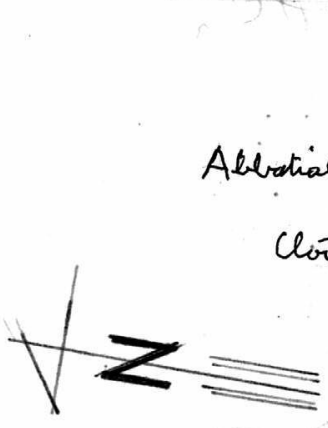

(àlétage)

Logís abbitial

Sozigravium

Infirmeric:

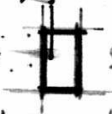

$\because$ Dortoir

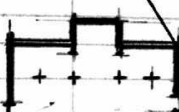

Salle capituluire

$\prod_{+++7}$

1

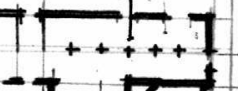

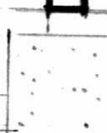

in

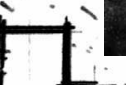

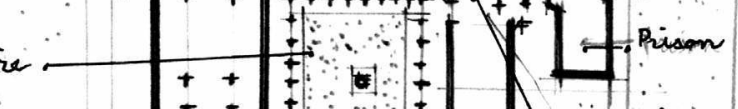

$t$

$+\frac{1}{+}+1$

commerno

Pigeonnior Bockangerie H1: D

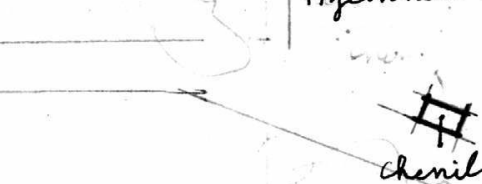

I

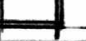

$\therefore$

effoin

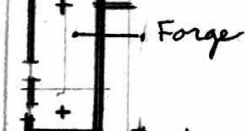

Plan d'organisation de l'abbaye cistercienne de Fontenay, Côte-d'Or, fondée par Bernard de Clairvaux. La Règle de saint Benoît comme instrument de programmation. 
prenant en charge des services publics comme les communes, responsables à partir de 1878 de l'édification des écoles. Il permet donc de planifier et de réaliser rapidement des opérations de construction à vaste échelle en en maîtrisant les coûts. Inspirés par les ordres religieux et militaires, les gouvernants laïcs, hommes d'État ou industriels du XIX $x^{e}$ siècle ${ }^{6}$, placent ainsi I'architecture au service de leurs objectifs politiques, sociaux et économiques selon des modalités qui mèneront progressivement au fonctionnalisme et à la standardisation des " process » et des éléments constructifs. La surface des locaux, la taille du mobilier et sa forme font l'objet de prescriptions très détaillées ${ }^{7}$.

Cependant, aux premiers plans-modèles, cahiers des charges comprenant des références quantitatives et des recommandations stylistiques illustrées par des dessins précis, succèdent des textes réglementaires définis en tant que programmes directeurs, comportant des schémas plus abstraits afin de ne pas trop contraindre l'invention formelle des concepteurs. Les effets de ce mode de programmation vont se traduire par la remarquable homogénéité architecturale des équipements publics construits à cette époque, qui n'empêcha toutefois pas l'affirmation d'une certaine singularité pour chaque édifice, à la différence du mouvement planificateur de l'après-guerre au $X X^{e}$ siècle. Autrement dit, le programme au XIX ${ }^{e}$ siècle explicite par des énoncés le "caractère ", le "type » dira-t-on plus tard, c'est-à-dire " la correspondance entre un espace projeté ou construit, et des valeurs sociales ${ }^{8}$ ", sans pour autant imposer une architecture totalement standardisée.

\section{Premières réflexions théoriques sur l'activité programmatique}

Si la notion de programme, comme outil de contractualisation, et celle de programmation, comme activité permettant de rationaliser les " process " de construction, s'affirment de façon assez précoce dans l'histoire de la production du cadre bâti, leur émergence ne s'accompagne pas toujours d'une réflexion théorique et méthodologique sur la façon de conduire l'activité programmatique. Ainsi, bien que le programme ait constitué très tôt un instrument pédagogique dans l'enseignement académique de l'architecture, il ne faisait pas pour autant l'objet d'un travail spécifique de la part des élèves afin de préciser les termes de la commande et servait seulement à leur mise en concurrence pour leur passage dans l'année d'étude supérieure.

Pourtantl'idée d'effectuer, pour chaque projetà réaliser, une étude fine des différents termes de la demande, n'est pas absente des premiers traités d'architecture en Europe, et notamment de celui d'Alberti dès la Renaissance. Dans De re aedificatoria, Leon Battista Alberti énonce un certain nombre de règles qui permettront à l'architecte de préciser la nature du programme qui lui est soumis à partir d'un dialogue à mener avec son commanditaire et d'un travail d'investigation personnel. Celles-ci sont guidées par trois dimensions censées être articulées tout au long du " process » de conception pour jouer le rôle de système opératoire. L'édification doit ainsi être envisagée par le concepteur du point de vue des besoins liés à la survie des hommes dans leur environnement (necessitas), des usages suscités par les désirs (commoditas) et de la beauté qui suscite le plaisir (voluptas) ${ }^{9}$.

\begin{abstract}
6. À cette même époque, dans le domaine privé, les ingénieurs chargés de la production du logement patronal relaient les mêmes types de préoccupations, de contrôle social et d'hygiène, comme le montre le cas du développement de la cité industrielle du Creusot analysé par Jean-Pierre Frey: La Ville industrielle et ses urbanités. La distinction ouvriers/employés. Le Creusot 1870-1930, Liège, Bruxelles, Pierre Mardaga, 1995.
\end{abstract}

7. Les travaux du Centre d'études, de recherche et de formation institutionnelle (Cerfi) puis ceux d'Anne-Marie Châtelet sur la production des équipements scolaires au XIX siècle et au début du XXe rendent compte de façon particulièrement éclairante de cette logique.

8. Christian Devillers, «Typologie de l'habitat et morphologie urbaine », l'Architecture d'aujourd'hui, n 174, juillet 1974, pp. 18-22.
9. Voir l'introduction de l'ouvrage Leon Battista Alberti. L'art d'édifier, traduit du latin, présenté et annoté par Pierre Caye et Françoise Choay, Paris, Le Seuil, coll. «Sources du savoir », 2004, p. 20. 
Quatre siècles plus tard, Eugène Emmanuel Violletle-Duc reprend dans ses Entretiens surl'architecture cette idée d'approfondissement des éléments du programme comme base de toute réflexion constructive. À l'instar de celle des théoriciens de son époque, la démarche de Viollet-le-Duc répond à un souci de rationalisation du processus de composition architecturale appréhendé depuis la fin du XVIII siècle selon le principe analyse/synthèse inspiré de la méthode cartésienne. Mais la sienne opère une rupture importante avec les doctrines rationalistes dominantes de son époque, dont Jean-Nicolas-Louis Durand est I'un des principaux instigateurs ${ }^{10}$. Alors que les enseignants des Beaux-Arts les moins académiques préconisent d'appliquer la raison cartésienne à la détermination des aspects techniques de la construction, la composition restant en revanche de I'ordre de l'intuition et du sens artistique, Viollet-le-Duc recommande d'y recourir pour l'ensemble du processus de conception, incluant alors un travail spécifique de programmation et redonnant à cette occasion une place prépondérante à l'utilitas, troisième pilier de la théorie vitruvienne. Pour lui, « les raisons premières, déterminantes en architecture » sont non seulement « les moyens matériels " mais aussi « le programme ${ }^{11}$ ". II distingue ainsi clairement le travail relevant de l'étude " des besoins » auxquels devait répondre l'édifice, de celui qui consiste à définir les matériaux et les techniques constructives adaptés.

On peut dire qu'Alberti et plus explicitement encore Viollet-le-Duc ont conféré une nouvelle dimension au programme, introduisant même la notion de programmation telle qu'elle se développera un siècle plus tard comme démarche de recueil, de traitement et d'analyse de données liés aux besoins ou aux désirs des hommes. En effet, si dans les termes de la relation entre le prince et I'architecte-tout comme dans l'enseignement classique de l'architecture qui cherchait à en reproduire les principes -, le programme correspondait à la partie donnée comme commande par le premier au second, Alberti et Viollet-le-Duc en ont fait le résultat d'un travail spécifique, contextualisé et rationnel, à la base de l'activité de conception.

Ces deux théoriciens apportent une autre contribution majeure concernant la définition du programme, répondant selon nous à une même préoccupation à plusieurs siècles d'écart. Dans son traité De re aedificatoria, Alberti montre que la conception des bâtiments peut être abordée de deux façons: soit en considérant que les édifices répondent, selon leur fonction générale, à des principes d'organisation et de construction universels, soit en estimant que chaque réalisation est particulière car la demande de chacun est singulière. Dans le premier cas, I'architecte peut se limiter à repérer dans les classifications existantes des édifices selon leur destination, leur caractère public ou privé, etc., les modèles qui sont les plus proches de l'objet à réaliser. Dans le second cas, il devra chercher à définir des principes constructifs singuliers adaptés à chaque projet. On retrouve dans cette double approche la question fondamentale du rapport entre l'universel et le particulier qui préoccupera jusqu'à la fin du $x^{e}$ siècle bon nombre de théoriciens de l'architecture et de l'urbanisme, et qui dans une réflexion sur la programmation doit faire l'objet d'une grande attention. Alberti propose
10. Jean-Nicolas-Louis Durand adopte une posture critique vis-à-vis de l'apprentissage par l'imitation des modèles d'édifices qui a marqué l'enseignement de l'architecture jusqu'alors. Pour lui, « avant de composer, il faut savoir avec quoi l'on compose; or la composition de l'ensemble de l'édifice n'étant que l'assemblage de leurs parties, il faut connaître celles-ci avant de s'occuper de l'autre. Or ces parties n'étant elles-mêmes qu'un composé des premiers éléments des édifices, [...] ces premiers éléments doivent être les premiers que I'architecte doit isoler » (J.-N.-L. Durand, Précis des leçons d'architecture données à l'École royale polytechnique, Paris, Chez l'auteur, 1809, p. 27-28). Les « premiers éléments » sont en fait les matériaux de construction. Viennent ensuite, dans ces chaînes de raisonnement, les formes et proportions (éléments de l'édifice) que l'on peut obtenir avec les divers matériaux, dont la composition conduit à la formation des parties de l'édifice qui, elles, servent enfin à la composition de l'ensemble de l'édifice.

11. Eugène Emmanuel Viollet-le-Duc, Entretiens sur l'architecture, dixième entretien, [Paris, Morel, 1863], rééd. Mardaga, 1986, p. 464. 
une démarche articulant ces deux dimensions: il fait « l'hypothèse selon laquelle les édifices, nécessairement différenciés par leurs conditions d'utilisation, n'en obéissent pas moins à des règles universelles ". II suggère ainsi d'aborder, dans un premier temps, les aspects qui sont communs et en quelque sorte intrinsèques à ce que l'on peut désigner comme des types d'édifices, puis d'apporter des adaptations en fonction de la singularité du contexte et de la demande ${ }^{12}$.

Cette distinction réalisée par Alberti entre ce qui relève du général ou du spécifique annonce celle qui est opérée par Viollet-le-Duc lorsque ce dernier fait référence à deux types de programmes : I'un correspondant à la vocation générale de l'édifice et exprimant des utilisations courantes censées être bien connues quel que soit le contexte, l'autre mettant en jeu des contingences singulières. Viollet-le-Duc apparaît à cette occasion dans la lignée d'Alberti, comme l'un des premiers théoriciens de l'architecture à avoir préconisé l'étude des nécessités et des pratiques sociales, civiles ou religieuses, dans le processus de conception en tant que démarche essentielle contribuant à l'élaboration d'un programme. II ne parviendra pas pour autant à imposer sa vision dans l'enseignement académique de l'architecture en France.

\section{La programmation en France dans les années 1960 ; entre souci de rationalisation des " process " et préoccupation d'innovation} La production urbaine d'après-guerre en France a souvent tendance à être abordée de façon monolithique car associée à la période économique des Trente
Glorieuses et à la réalisation des grands ensembles. Cependant, si l'on s'intéresse à ses modalités d'organisation et aux rapports existant entre planification et programmation, on est conduit à distinguer plusieurs phases. À une phase dite de Reconstruction, assez chaotique, succède à partir de la fin des années 1950 une période de mise en place de tout un ensemble de structures financières et techniques mais aussi de création de nouvelles procédures opérationnelles. La notion de "programmation urbaine " s'impose à la fin des années 1950 dans un contexte général de rationalisation des processus de planification économique et budgétaire dont elle résulte autant dans ses finalités que dans ses principes méthodologiques. Le Commissariat général du Plan entreprend en effet de soutenir la croissance en diffusant à tous les niveaux de l'administration française des méthodes de gestion prévisionnelle s'appuyant sur le cadre de la Comptabilité nationale. Ce que l'on appelle l'esprit du Plan se caractérise par l'incitation faite aux agents publics et privés de définir conjointement leurs actions dans la perspective de préparer I'avenir tout en mettant en cohérence des politiques publiques à différentes échelles territoriales et temporelles. Concernant I'aménagement de l'espace, cette logique se traduit à partir de la seconde moitié des années 1950 par l'affirmation d'un principe de programmation urbaine. Cette expression est alors utilisée pour marquer une volonté de mieux organiser le " phénomène d'urbanisation » et de mieux articuler les différentes échelles de l'aménagement, du national au local, du long terme au moyen terme, ce qui n'avait pas été le cas au cours de la Reconstruction. 
Alors que la notion de planification concerne la politique d'aménagement à l'échelle nationale puis régionale, la programmation urbaine est censée en être la déclinaison pour une agglomération, une commune ou un quartier. Cette notion est définie par rapport à deux grandes catégories d'opérations: les opérations d'infrastructures, qui se divisent elles-mêmes en opérations d'urbanisme de plusieurs natures, et les opérations dites de superstructures (équipements scolaires, sportifs, administratifs, sociaux, commerciaux...). Pour mettre en place cette programmation, deux dispositifs à vocation différente sont créés: les Zones à urbaniser par priorité (ZUP) et les Programmes de modernisation et d'équipement (PME). La logique des PME consiste à intégrer la programmation urbaine dans des perspectives de planification économique et d'aménagement du territoire fixées à l'échelon national et régional. Les PME visent les agglomérations ou les villes moyennes connaissant une forte croissance. Ils sont établis en phase avec la planification nationale, qui fixe alors des objectifs de croissance économique selon une logique volontariste. Ils doivent préciser la hiérarchie des urgences et des investissements à réaliser sur la durée couverte par le Plan, en donnant aussi des objectifs à "l'horizon de dix ans ». Les modalités de répartition des équipements collectifs sur le territoire sont censées s'appuyer sur un système de répartition organisé par la grille dite Dupont ${ }^{13}$, tandis que la définition même des caractéristiques de chaque équipement est soumise à des référentiels élaborés de façon sectorielle par les différentes autorités de tutelle.

Tirant les enseignements des premiers PME réalisés au cours des III et IVe Plans, le Commissariat général du
Plan prodigue au milieu des années 1960 de nouvelles recommandations sur leur contenu et leur mode d'élaboration. Face à des déséquilibres territoriaux qui s'accentuent, il incite à une plus grande prise en compte des spécificités économiques et démographiques locales dans l'aménagement des espaces urbains, et à la conduite de démarches fondées sur la prévision et la prospective grâce à la réalisation d' « études préalables » et à la définition d'hypothèses de développement à différentes échelles spatiales et temporelles. Basées sur l'organisation de dispositifs de concertation entre forces vives, la planification et la programmation urbaines prennent ainsi une dimension plus stratégique dans le sens où elles commencent à être envisagées comme des démarches permettant de prendre des décisions à partir de l'étude de scénarios ${ }^{14}$. La politique de mise en œuvre de vastes programmes d'équipements se trouve dès lors prise dans une double logique. D'une part, la volonté de déployer une planification urbaine plus stratégique et une politique de rééquilibrage régional invite à mieux tenir compte des spécificités locales. D’autre part, l'ampleur et l'urgence des problèmes d'équipements à traiter encouragent des modes de planification et de programmation basés sur la rationalisation des procédures, la simplification des programmes et l'uniformisation des produits.

Bien qu'extrêmement dépendantes des décisions des administrations centrales pour l'attribution de subventions, certaines collectivités locales, des villes moyennes soucieuses de se dégager des logiques procédurales étatiques, s'engagent dans des démarches de programmation urbaine singulières avec l'aide
13. Cette grille, officialisée en 1958 par le ministère de la Construction, suite au rapport de Gérard Dupont sur les équipements résidentiels, devait permettre de définir la nature, la taille, la localisation, le coût et l'échéancier des programmes d'équipements à réaliser, à partir du nombre d'habitants et de logements escompté dans l'aire d'affluence. Elle laissait en revanche les ministères concernés libres de fixer les caractéristiques architecturales de ces équipements. Cette grille sera réactualisée en 1961 et en 1965, sans jamais véritablement réussir à être totalement suivie. Les systèmes de répartition normatifs furent en effet sans cesse contestés par la prégnance des « jeux locaux », c'est-à-dire des systèmes d'influence entre élus et représentants de l'État.

14. Ce type d'approche s'inscrit plus généralement dans une nouvelle logique de définition de l'action publique à travers la mise en place d'une réforme de la gestion du budget de l'État qu'incarne la politique de Rationalisation des Choix Budgétaires (RCB) instituée à partir de 1968 et expérimentée dans plusieurs administrations centrales à l'incitation du ministère de I'Économie et des Finances. La RCB s'inspire du Planning Programming Budgeting System américain, mais résulte avant tout de réflexions et d'expériences menées sur le calcul économique depuis une quinzaine d'années en France, par le Commissariat général du Plan et par Électricité de France. 


\section{GRILLE D'ÉQUIPEMENT}

\section{D’UN GRAND ENSEMBLE D’HABITATION}

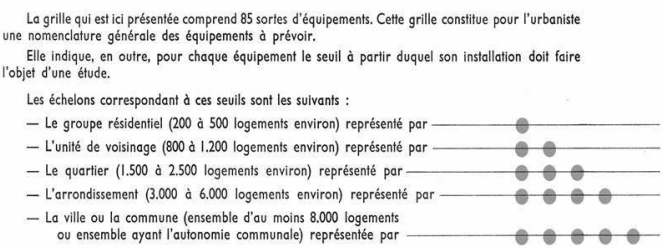

I. - Equipement Scolaire, Culturel et Cultuel.

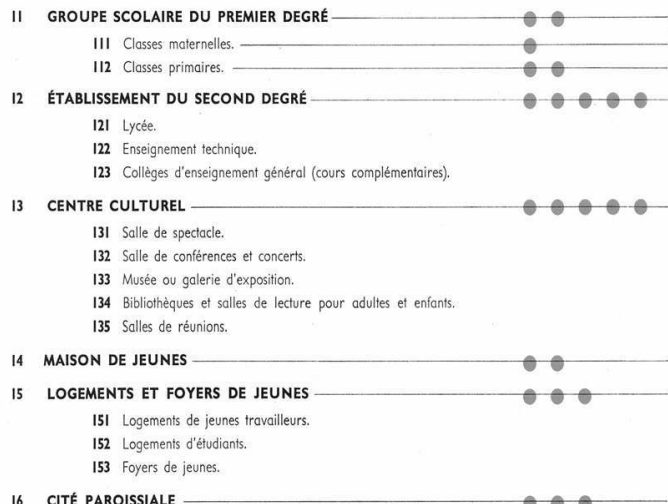

\section{II. - Équipement Commercial.}

$\begin{array}{lll}21 & \text { CENTRES SECONDAIRES } \\ 22 & \text { CENTRE PRINCIPAL } \\ 23 & \text { HOTELLERIE }\end{array}$

\section{III. - Equipement Social et Sanitaire.}

31 ÉQUIPEMENT SOCIAL

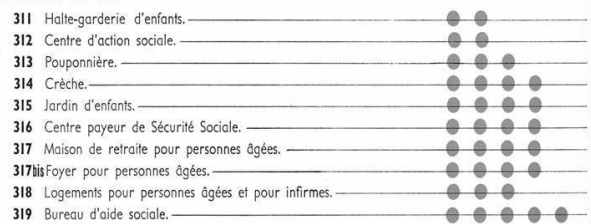

32 ÉQUIPEMENT SANTÉ

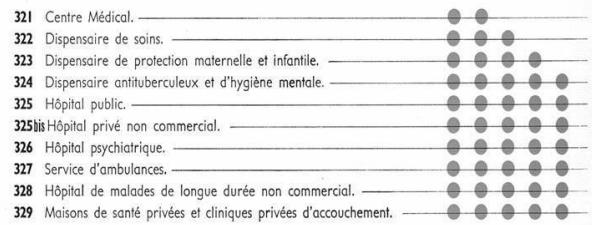

Un outil de programmation urbaine

normative : la grille dite Dupont,

Urbanisme, n $62 / 63,1959$, p. 22-23.

\section{IV. - Espaces plantés, Parkings et Sports.}

41 JARDINS D'IMMEUBLES

411 Zones de pré-habitation

412 Aires de jeux libres

413 Espaces clos pour enfunts.

414 Cadre de verdure.

42 PARKINGS, VOIES ET PLACES PLANTEEES

421 Parkings.

422 Voies et places plantées.

43 PARCS ET JARDINS PUBLICS

431 Jardins publics.
432 Parcs urbains.
433 Grand parc boisé
INS DE CULTURES INDIVIDUELLES
EEMENT SPORTIF NON SCOLAIRE
451 Terrains de volley-ball et bosket-ball.
452 Courts de tennis.
453 Terrains d'entrainement pour le football, le rugby et le hand-ball.
454 Terrains ou stede de compétition omnisports.
455 Salle de sports.
456 Bassin de natation.
456 bis Piscine couverte.

46 CIMETIÈRE

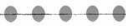

\section{V. - Equipements généraux.}

51.7 ADMINISTRATION MUNICIPALE OU D'INTÉRÊT LOCAL

5 II Services administratis municipoux.

512 Services techniques municipaux (Direction ef Bureau d'études)

513 Bureaux de la Municipalité ef locaux de cérémonie.

514 Syndicat d'initiative.

515 Bureaux des organismes constructeurs.

52 INSTALLATIONS ANNEXES DES SERVICES TECHNIQUES D'INTERET LOCAL

$$
521 \text { Abattoirs. }
$$

522 Ateliers municipaux (garcges, réserves, entretien).

523 Eav ef assainissement (usines et stations).

524 Entretien de la voirie et des espaces plantés (parc à matériel, pépinieres).

525 Enlèvement des ordures ménagères (garoges et usine de traitement)

526 Pompes Funébres (garages et magasins).

527 Chauffage urbain.

53 POLICE INCENDIE

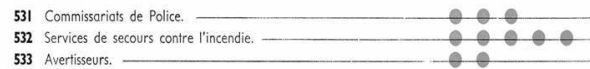

533 Avertisseurs.

54 ÉQUIPEMENT DE LA VOIE ET DES ESPACES PUBLICS

541 Eclairage publect

542 Bancs, boites à papier, plans de la ville, plaques indicatrices, panneaux d'affichage, etc.

543 Parkings publics.

55 SERVICES D'ÉTAT

551 Gendarmerie.

552 Direction des Impôts

553 Recette-Perception

56 P. T. T.

561 Bureau de poste principal

563 Central téléphonique.

564 Bottes cux letires.

565 Cabines de téléphone public.

0.00

UNICATIONS

571 Stations cutobus.

572 Gare routière,

573 Station S. N. C.

58 MARCHES PUBLICS

000 
d'agences d'urbanisme que certaines créent ou de structures parapubliques ou privées ${ }^{15}$ qui se mettent à développer aussi bien des activités d'études que de recherche. On remarque alors que les professionnels faisant de la programmation dans ces organismes ont le plus souvent des formations en économie, droit, sciences politiques, géographie, sociologie. Ils se désignent rarement comme programmeurs ou programmateurs ${ }^{16}$, bien que le terme existe à cette époque dans la logique administrative, mais se réfèrent à leur discipline d'origine ou revendiquent le statut d'urbanistes. Ils se trouvent de ce fait dans une situation conflictuelle avec les architectes de la Société française des urbanistes, qui ont monopolisé le titre jusque-là. Leur posture est résolument militante. Ils défendent de nouvelles pratiques de l'urbanisme, s'appuyant sur des principes de travail collectif, de pluridisciplinarité des équipes et de participation des habitants, sur fond de dénonciation du centralisme bureaucratique et technocratique, ou encore des méfaits de l'économie libérale. Les plus critiques dénoncent le fonctionnalisme du Mouvement moderne et les approches normatives des administrations centrales ou déconcentrées basées sur des analyses de besoins préformatés ${ }^{17}$. Ils remettent également en cause l'approche techniciste et modélisatrice des ingénieurs des Ponts, corps qui a pris une position dominante à la suite de la création du ministère de l'Équipement. Dans le domaine de la programmation architecturale, on note que les premiers bureaux d'études privés proposant explicitement ce type d'expertise présentent des équipes pluridisciplinaires dans lesquelles sociologues et psychologues jouent un rôle important en raison du souci de ces structures d'aborder la programmation des équipements publics par des réflexions sur des aspects organisationnels.

Les bureaux d'études préopérationnelles qui se développent dans la deuxième partie des années 1960 sont à I'origine d'innovations importantes en matière de démarches et de définition d'outils programmatiques qui trouveront leur application jusqu'au milieu des années 1970 dans l'aménagement des quartiers périphériques des villes moyennes et des villes nouvelles, ainsi que dans la promotion des équipements intégrés. Soutenues pour certaines par l'État, ces expérimentations ne remettront pas pour autant fondamentalement en question la prédominance des approches technicistes dans le domaine de l'aménagement, ni le recours massif aux référentiels normatifs dans celui du logement et des équipements publics, mais elles donneront une nouvelle dimension à l'approche de la programmation.

\section{La réforme de l'ingénierie publique de 1970 à 1990 ; la consommation de la rupture entre programmation et conception}

Au cours des années 1970, I'autonomisation de la programmation dans la démarche de projet s'officialise en France à l'occasion d'une réforme de l'ingénierie publique soutenue par des objectifs politiques, économiques et techniques. La réalisation du Centre Georges Pompidou à Paris, dans le quartier des Halles, a préparé cette réforme qui trouve ses premières grandes applications à l'occasion de la réalisation des villes nouvelles. Jusqu'au début des années 1970, les architectes chargés de réaliser des édifices publics en France intervenaient
La traduction française du PPBS insiste sur la notion de choix que la méthode doit chercher à éclairer, en anticipant et en explicitant les conséquences des moyens par rapport aux buts visés par des démarches d'évaluation ex-ante ou ex-post. Les travaux des théoriciens de la décision sur la rationalité limitée, comme ceux d'Herbert Simon, seront au cœur de cette approche.

15. Telles que le Bureau d'études et de réalisations urbaines (Beru), le Bureau d'études pour l'urbanisme et l'équipement (Beture) et le Centre d'études et de recherche sur l'aménagement urbain (Cerau), ou dans le privé l'Atelier d'urbanisme et d'architecture (AUA). Voir sur ce sujet Viviane Claude, Faire la ville. Les métiers de l'urbanisme au XX siècle, Marseille, Parenthèses, coll. «Eupalinos », 2006, 253 pages.

16. Programmeur, programmateur, programmiste: tels sont les différents noms qui ont été successivement attribués en France aux professionnels de la programmation. Leur apparition dans le langage commun correspond à des périodes que l'on peut grosso modo identifier, mais aucun d'entre eux ne s'est à ce jour complètement imposé. Le premier, et sans doute le plus ancien, a semble-t-il commencé à être utilisé à partir des années 1950. Il est alors employé aussi bien pour les professionnels de l'aménagement de l'espace que pour les informaticiens.

À partir de la fin des années 1960, celui de 
aussi régulièrement dans la définition du programme aux côtés d'un représentant de l'État et des services techniques concernés. Sur la base des normes générales éditées par le ministère de tutelle concerné, le commanditaire affinait le programme de son équipement avec l'architecte qu'il avait désigné sur liste d'agrément. À partir de 1973, le rôle et les relations entre commanditaires et maîtres d'œuvre commencent à profondément se transformer. Les directives réglementaires adoptées à cette date interviennent dans un contexte marqué par deux, voire trois phénomènes essentiels. L'un d'eux est l'amorce d'une décentralisation progressive des compétences et d'une diversification des acteurs de l'aménagement au niveau local. Il est de plus en plus admis dans ce contexte que les conditions de passation des marchés aux concepteurs sont loin d'être satisfaisantes et demandent à être clarifiées: la rémunération des architectes étant jusqu'alors proportionnelle au coût de l'ouvrage, cela les incitait à engager des travaux dispendieux. Dans une conjoncture économique qui se dégrade, le souci de maîtrise des budgets devient plus important. Un autre phénomène est la prise de conscience collective, au sein même des instances de l'État, des conséquences préjudiciables qu'ont eues les modes d'urbanisation et notamment de production des grands ensembles lors des décennies précédentes. L'absence de prise en considération des modes de vie des habitants et la mauvaise qualité des constructions commencent à être dénoncées. Un changement dans les processus d'ingénierie est réclamé avec deux idées centrales: mieux préparer la définition de la commande et mettre en concurrence les maîtres d'œuvre.

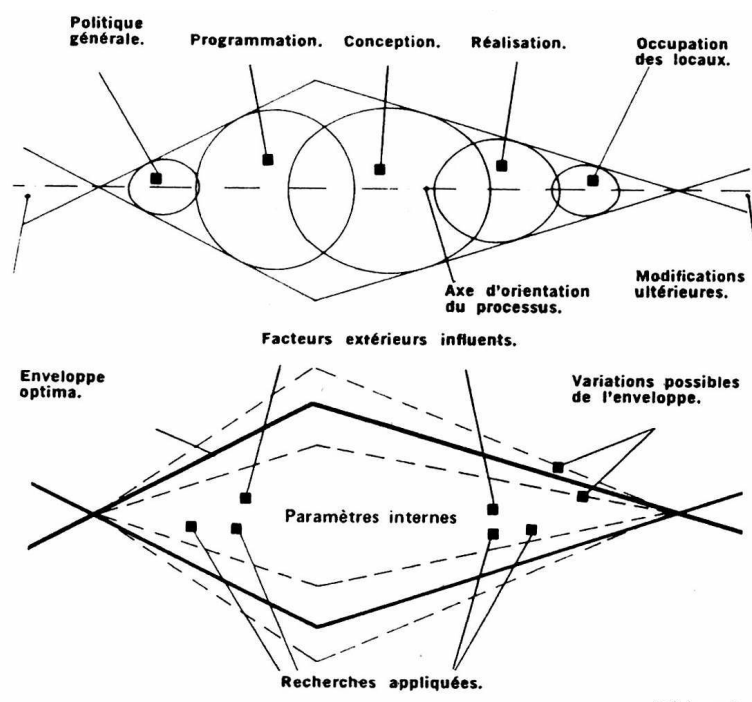

Schéma 1.
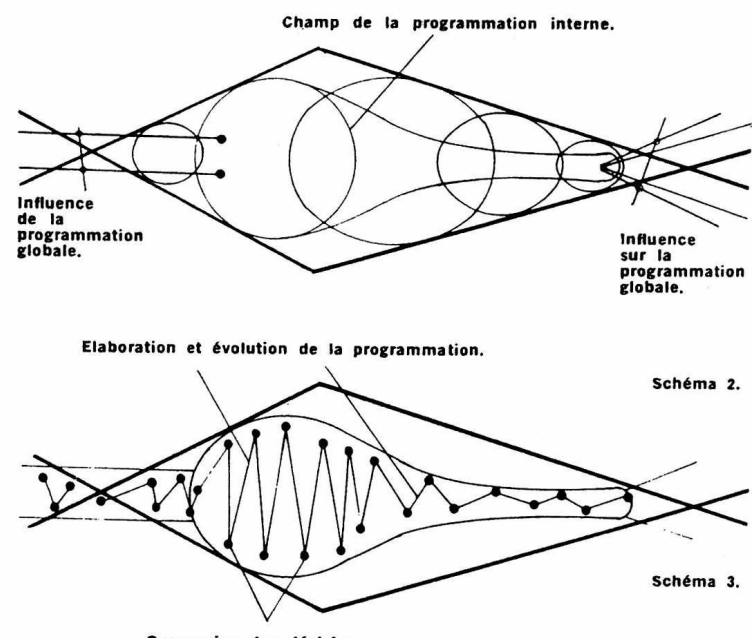

Succession des décisions. programmateur l'a peu à peu éclipsé. Depuis le début des années 2000, le nom de programmiste a été officiellement retenu par le Syndicat des programmistes en architecture et en aménagement (SYPAA).

17. Certains privilégient alors d'autres types d'approches visant à analyser les comportements des agents économiques afin de mieux envisager les mécanismes de la décision, ou encore à mener des enquêtes auprès des habitants pour mieux connaître
La démarche programmatique conceptualisée par François Lombard, à l'occasion de la réalisation du Centre Georges Pompidou. Extraits parus dans "Conception architecturale et démarche programmatique », Techniques et architecture, $n^{\circ} 303$ mars 1975 , p. 55-59. a. Réalisateur.

b. Utilisateur.

c. Programmateur.

d. Concepteur.

e. Maitre de l'ouvrage.

2. Mise à disposition des locaux.

3. Exécution.

4. Conception.

5. Programmation

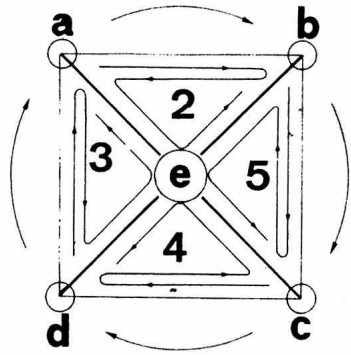


Les dispositions de la directive du 8 octobre 1973 entérinent officiellement une seconde autonomie de la conception ${ }^{18}$. Elles dissocient nettement les missions du maître d'ouvrage de celles du maître d'œuvre en faisant de l'élaboration du programme une prérogative strictement attribuée au premier. La directive insiste sur l'importance de prendre en considération le contexte d'un projet; elle montre ainsi une volonté de nuancer la logique planificatrice centralisée uniformisant les problématiques constructives. Mais elle continue néanmoins d'exprimer une approche très technico-administrative de la programmation, centrée sur la constitution d'un programme-cahier des charges rassemblant des éléments essentiellement quantifiables, techniques ou réglementaires. Le terme de programmation n'est pas employé dans ce texte; la notion de document programme est en revanche sans cesse privilégiée. Celui-ci est présenté comme le résultat d'une procédure cadrée par une série d'études préalables au travail de conception. Une fois devenu un cahier des charges permettant de passer commande à un maître d'œuvre, le programme n'est plus mentionné, comme s'il ne pouvait plus évoluer.

Alors que les textes de 1973 défendent l'utilité du programme surtout par rapport à une nécessité de contrôle des dépenses, la loi sur la maîtrise d'ouvrage publique du 12 juillet 1985 régissant les relations entre maîtrise d'ouvrage publique et maîtrise d'œuvre privée va renforcer ce principe mais en défendant aussi plus explicitement un objectif de promotion de la qualité des constructions. La loi MOP intervient dans un contexte d'entrée en vigueur des lois de décentralisation. L'une des intentions majeures de ce texte est de renforcer la responsabilité des maîtres d'ouvrage pour les impliquer davantage dans les résultats attendus de leurs projets. Ils sont ainsi contraints de s'assurer de la faisabilité et de l'opportunité de l'opération, d'en déterminer la localisation, d'en définir le programme et de réaliser en amont des estimations fiables sur le « coût d'objectif » des opérations comprenant à la fois la rémunération de la maîtrise d'œuvre et le coût des travaux. Dans ce contexte d'augmentation de son niveau de responsabilité et de complexification des procédures, le maître d'ouvrage doit se doter de nouvelles compétences. Il peut ainsi « confier les études nécessaires à l'élaboration du programme et à la détermination de l'enveloppe financière prévisionnelle à une personne publique ou privée » (article 2). Cet article sera fondamental pour beaucoup d'architectes qui, dans une période de forte crise de leur profession, y percevront un encouragement à se spécialiser dans cette activité. II marque d'une façon générale l'amorce d'une véritable professionnalisation de la fonction de programmation.

La loi, comme ses décrets d'application publiés en 1993, ne définit cependant toujours pas la notion de programmation, terme qu'elle n'utilise en fait pas, mais donne des indications sur le contenu du programme. Elle précise que l'élaboration de ce document doit être précédée d'études sans mentionner toutefois si celles-ci font partie intégrante d'une démarche de programmation. Si la loi MOP continue de procéder à un découpage précis et assez normatif du processus projet par une suite d'études aux intitulés prenant une valeur réglementaire, elle enrichit néanmoins, par son article 2 leurs véritables aspirations ou désirs. Ces professionnels des bureaux d'études prendront appui sur les travaux menés par l'équipe de Paul-Henry Chombart de Lauwe dès la fin des années 1950 ainsi que sur ceux du Centre de sociologie urbaine (CSU), du Centre d'études, de recherche et de formation institutionnelle (Cerfi), ou encore sur ceux de la Compagnie des économistes et des psychosociologues (CEP).
18. La première ayant été opérée à la Renaissance, entre le travail de conception de celui d'exécution. 
(alinéa 3), les attendus relatifs au programme en leur donnant un caractère plus dynamique et qualitatif par rapport aux directives précédentes. La notion d'objectifs est mentionnée et précède à présent celle de besoins. Les contraintes et exigences à définir ne sont plus seulement d'ordre technique et financier, elles doivent aussi s'exprimer en termes de « qualité sociale, urbanistique, architecturale, fonctionnelle, d'insertion dans le paysage et de protection de l'environnement, relatives à la réalisation et à l'utilisation de l'ouvrage ". Cinq ans après la création du Plan construction et architecture, celle de la Mission interministérielle pour la qualité des constructions publiques, en 1977, avait confirmé le souci de l'État de montrer qu'il souhaitait rompre avec les approches productivistes de l'aprèsguerre. Sous l'impulsion de la MIQCP, la loi MOP présente donc clairement le programme comme « le lieu où commence le cheminement de la qualité ». Si la loi MOP donne une vision plus large des attendus de la programmation que la directive de 1973, elle entérine l'autonomisation de la programmation par rapport à la conception en confortant une forte logique séquentielle dans les processus de projet ${ }^{19}$, allant de pair avec un mouvement général de division technique du travail particulièrement important dans le domaine de la construction $^{20}$.

Tout en ayant eu le mérite de responsabiliser fortement les maîtres d'ouvrage par rapport à la définition de leurs intentions, la loi MOP a donc dissocié de façon importante la programmation du travail de conception. Ce phénomène contribuerait à expliquer que la plupart des écoles d'architecture en France ignorent ou margi- nalisent dans leurs cursus l'enseignement de la programmation, comme si cette activité n'avait rien à voir avec celle du " projet » et était seulement une préoccupation des maîtres d'ouvrage. Les guides sur la programmation publiés depuis les années 1980, à l'initiative d'administrations centrales, sont d'ailleurs principalement orientés vers les maîtres d'ouvrage et leurs assistants. Ils ont aussi tendance à définir cette activité prioritairement par rapport au cadre légal et donnent du coup souvent à cette démarche un caractère assez procédural. Les théoriciens anglo-saxons du Design qui s'intéresseront à cette activité développeront une tout autre approche à cet égard, envisageant la programmation comme une méthode heuristique aidant à la définition et à la résolution de problèmes de conception. Enfin, on remarquera que la loi MOP a été élaborée essentiellement à partir d'une réflexion sur les projets de construction, alors qu'elle s'applique également aux opérations d'urbanisme où la coupure entre programmation et conception est en général difficile à établir de façon aussi séquentielle. Le recours de plus en plus important à la procédure des marchés d'études de définition simultanés à partir des années 1990 s'expliquera en partie par le souci de retrouver une meilleure articulation entre ces deux activités dès l'amont des projets. Les recherches-expérimentations menées par le département de sciences humaines du CSTB sous la direction de Michel Bonetti et Michel Conan sur « la programmation générative ", à la fin des années 1980 et au début des années 1990, auront aussi pour objectif de remettre en question la forte dissociation existant entre ces deux activités.
19. Cette logique est dictée par une représentation rationaliste de la construction de la décision publique qui continue de dominer dans l'administration française malgré l'expérience de la RCB. Lucien Sfez, La Décision, PUF, coll. "Que-sais-je? », 1984, mis à jour en 2004, 128 pages.
20. François Lautier, « La situation française: manifestations et éclipses de la figure du maître d'ouvrage ", in F. Lautier (sous la responsabilité scientifique de), Les Maîtrises d'ouvrage en Europe: évolution et tendances, vol. IV de la série L'Élaboration des projets architecturaux et urbains en Europe, Paris, Puca, 2000, p. 15-23. 


\section{Une approche de la programmation aujourd'hui en tension entre dimensions stratégiques et dimensions techniques}

"L'an I de la programmation remonte en France aux années soixante-dix, il y a donc bientôt 30 ans ", pouvaiton lire en éditorial de la première lettre publiée par I'Institut de programmation en architecture et en aménagement (IPAA), en novembre 1994. Cette affirmation illustre bien la place et l'impact qu'ont eus pour les professionnels de la programmation architecturale la réalisation du Centre Georges Pompidou et la réforme de l'ingénierie publique, auxquelles il est fait ici principalement référence. Or, si un processus de professionnalisation relatif à l'exercice de cette activité s'est bien affirmé en France à la suite de ces deux événements et lors de la création des villes nouvelles, nous avons souligné que des pratiques de programmation avaient été institutionnalisées et s'étaient développées dès le début des années 1960 dans le domaine de l'aménagement urbain. L'omission des promoteurs de I'IPAA n'est pas fortuite et témoigne d'une représentation assez partagée chez les professionnels de la programmation patentés que nous avons interrogés depuis une dizaine d'années, qui, pour la plupart, ont commencé à exercer à l'échelle architecturale.

En fait, si l'activité de programmation s'est clairement singularisée dans le domaine des constructions publiques avec les décrets sur l'ingénierie publique et la systématisation des concours d'architecture, il n'en a pas été de même à des échelles d'aménagement plus importantes. La fonction de programmation en urbanisme telle qu'elle s'était affirmée au cours des années 1960 s'est trouvée remise en question au cours de la décennie suivante, aussi bien dans sa dimension rationaliste que dans sa dimension plus stratégique, dans un contexte général de critique des dispositifs de planification technocratique, de décentralisation des compétences et de multiplication des incertitudes liées à la faisabilité des opérations. Elle perd alors de sa visibilité avec la prédominance d'une logique dite opérationnelle portée par des structures techniques d'aménagement intervenant pour le compte des collectivités locales, puis, à partir de la fin des années 1980, avec l'avènement d'une approche du projet urbain centrée sur un souci de la forme urbaine portée par les architectes-urbanistes. Elle se fragmente en une multitude d'études dites préalables et, dans sa dimension la plus repérable, se voit restreinte à une activité d'élaboration de programmes-cahiers des charges mettant surtout en jeu des aspects techniques et financiers. Avec le souci de développer des démarches de programmation-conception plus itératives depuis dix ans, elle est aussi partiellement prise en charge de façon implicite par les concepteurs urbains, souvent au prix d'une certaine déresponsabilisation des maîtres d'ouvrage. L'utilisation de la procédure des marchés de définition est particulièrement révélatrice de cette tendance où, sous couvert de stimuler l'invention et de favoriser une plus grande interaction entre programmation et conception dès l'amont des projets, nombre de maîtres d'ouvrage se sont mis à attendre des maîtres d'œuvre des solutions à des problèmes qu'ils n'osaient pas poser ${ }^{21}$.

Cependant, avec l'obligation actuelle de mise en œuvre de dispositifs de coopération entre acteurs et de

21. Jodelle Zetlaoui-Léger, «Marchés de définition simultanés : un succès à interroger », Urbanisme, $n^{\circ} 325$, juillet-août 2002, pp. 14-17. 
SCHEMA GENERAL DE COORDINATION DES INSTANCES

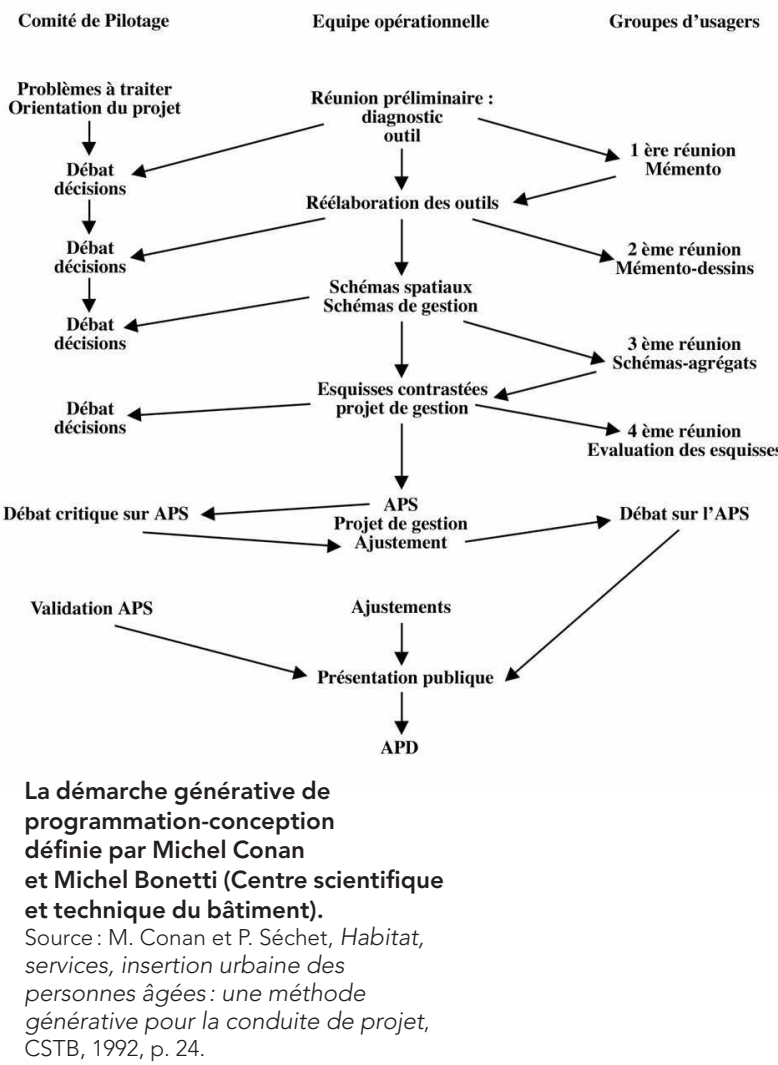

prise en considération des problématiques d'usages et de gestion tout au long d'un projet dans une perspective d'aménagement durable, une nouvelle demande d'assistance à maîtrise d'ouvrage en programmation paraît émerger. Elle ne vise plus seulement à réaliser des études préalables ou à rédiger des programmes mais à organiser des démarches de prise de décision et de concertation, y compris avec les citoyens-usagers. Elle s'inscrit dans un processus de coproduction du sens d'un projet, dimension qui a été largement déléguée ces dernières années aux logiques de marché et aux concepteurs urbains. Par ailleurs, s'il existait jusqu'à récemment un certain cloisonnement entre programmation architecturale et programmation urbaine dans les pratiques comme dans les représentations, les frontières paraissent aujourd'hui beaucoup plus floues: la nécessité de repositionner des projets d'équipements dans des dispositifs de politiques publiques plus globaux conduit manifestement de plus en plus de programmistes autrefois centrés sur l'échelle architecturale à repositionner leurs compétences dans une perspective plus large, le projet de construction se révélant être pour partie un projet d'urbanisme.

La teneur de ces tendances et leurs incidences sur la nature des projets réalisés mériteront d'être étudiées de façon précise dans l'avenir selon les échelles d'intervention, les formes d'organisation des maitrises d'ouvrage et les secteurs d'activité concernés. Les différentes recherches et études que nous avons menées ces dernières années nous ont néanmoins déjà montré qu'audelà des diverses pratiques et représentations qui caractérisent aujourd'hui la programmation, deux logiques continueraient nettement de s'opposer. L'une consiste à 
I'envisager comme une démarche stratégique d'aide à la décision guidée par quelques grands principes générateurs mais qui s'organise de manière singulière en fonction des contextes et des intérêts en présence. L'autre, à laquelle elle est plus traditionnellement associée et que nous qualifierons de « techniciste » et normative, vise à la considérer comme une démarche de traitement systématique de données faisant l'objet d'un protocole prédé- terminé, linéaire, mettant en jeu des réponses prédéfinies. L'existence de ces deux idéaux-types peut être considérée comme une sorte de déclinaison particulière de la métaphore de la règle et du modèle qu'employait Françoise Choay il y a vingt-cing ans pour qualifier les deux postures qui ont structuré les premières théories de l'urbanisme. Elle renvoie aujourd'hui plus que jamais aussi à des enjeux de gouvernance spécifiques.

\section{La démarche de programmation concertée et participative (PCP)}

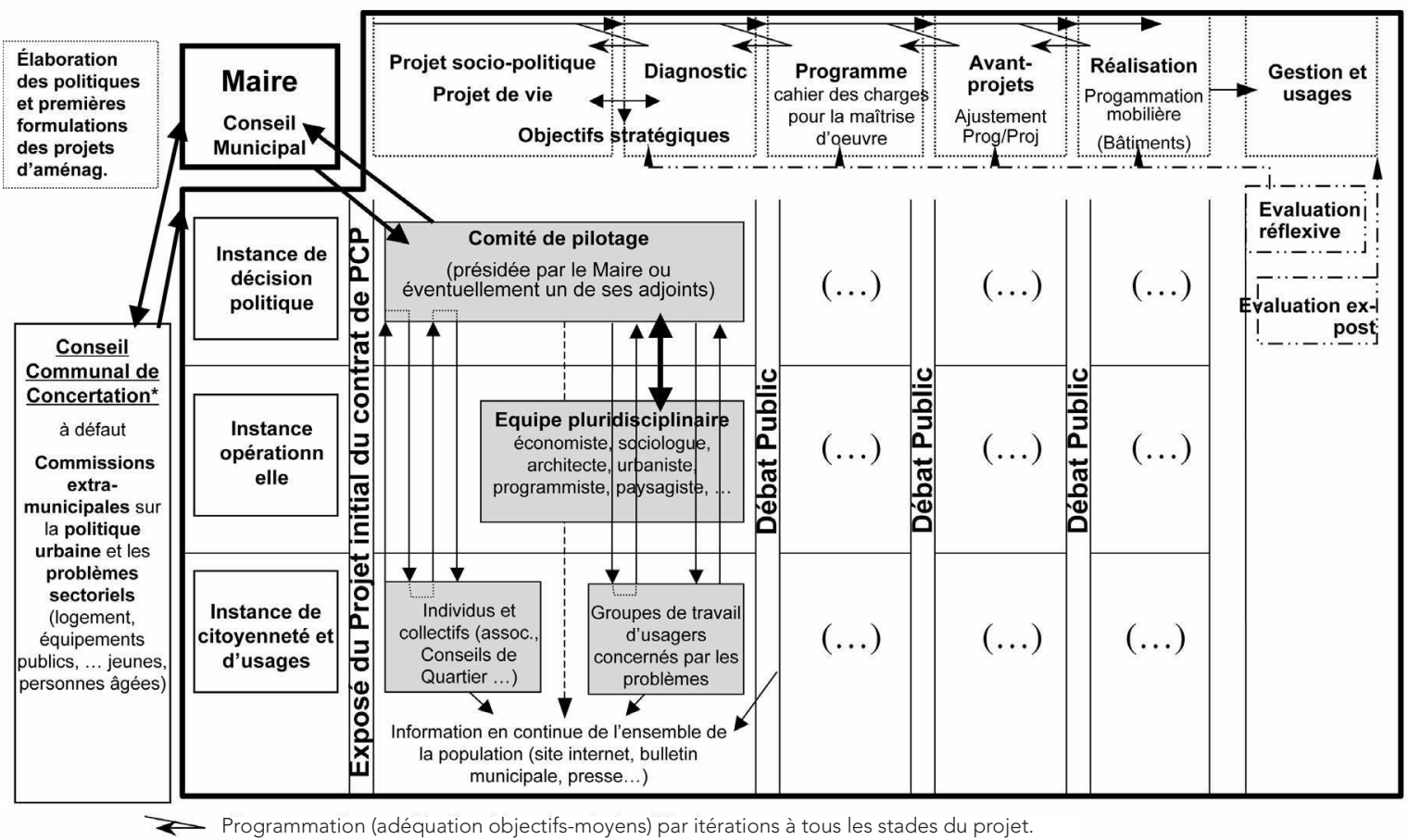

Principes d'une démarche de programmation citoyenne pour les projets d'urbanisme.

Source : P. Diméglio et J. Zetlaoui

en coll. avec F. Trichet, "Contribution

à l'élaboration d'une Charte de programmation

concertée et participative », EPPPUR-IUP :

http://www.univ-paris12.fr/iup. 